\title{
Early stoppage of antibiotics in blood culture negative term suspected early onset sepsis and their 7 days outcome
}

\author{
Upadhyay M.R. ${ }^{1}$, Pankaj K.C. ${ }^{2}$, Ganguly S. ${ }^{3}$, Murmu M.C. ${ }^{4}$ \\ ${ }^{1}$ Dr. Manas Ranjan Upadhyay, Assistant Professor, ${ }^{2}$ Dr. Kiran Chandra Pankaj, Resident Physician, ${ }^{3}$ Dr. Sristi Ganguly, \\ Resident Physician, ${ }^{4}$ Dr. Mangal Charan Murmu, Associate Professor; all authors are affiliated with Department of \\ Paediatrics, S.C.B. Medical College, Cuttack, Odisha, India.
}

Corresponding Author: Dr. Mangal Charan Murmu, Associate Professor; Department of Paediatrics, S.C.B. Medical College, Cuttack, Odisha, India. E-mail: mangal74murmu@yahoo.co.in

\begin{abstract}
Introduction: Sepsis is the commonest cause of neonatal mortality; it is responsible for about $30-50 \%$ of the total neonatal deaths in developing countries. It is estimated that up to $20 \%$ of neonates develop sepsis and approximately $1 \%$ die of sepsis related causes. Despite receiving extensive care in hospitals in the form of ventilator and inotropic support in intensive care set up, $2-3 \%$ of term and $20-30 \%$ of preterm infants still die of early onset sepsis (EOS). Clinical suspicion therefore frequently leads to empirical antibiotic therapy in uninfected infants. Treating an uninfected infant for 5 to 7 days means disrupting maternal bonding and breast feeding for an extended period of time, pain and distress from starting IVs, exposing the infant to drugs with potential toxicities, fostering the development of antibiotic resistant flora, and increasing the probability that the infant will experience a more serious morbidity later in the course of hospitalization. Material and Method: The study cohort consisted of the all term babies hospitalised with early onset sepsis to the S.C.B M.C.H and S.V.P.P.G.I.P, Cuttack from October 2016 to September 2018. Result: Out of total 186 neonates with suspected early onset sepsis, majority were male $(64 \%)$ and $93 \%$ presented within 24 hours of life. The most common perinatal risk factor noted was low birth weight (43\%) followed by perinatal asphyxia $(24.2 \%)$. $96.2 \%$ of the neonates were symptomatic at the time of admission; the most common symptom being respiratory distress (73.6\%). The blood culture was positive in $25.8 \%$ babies. The incidence of culture positive sepsis in symptomatic babies was $27 \%$. None of the asymptomatic babies were culture positive. Only $49 \%$ of screen positive babies were blood culture positive. Antibiotics were stopped in 133 babies and unnecessary use of antibiotics was reduced by $82.6 \%$. No sepsis related adverse outcome was noted in 7 days following stoppage of antibiotics. Conclusion: It is safe to stop antibiotics in term EOS babies if the 48 hour blood culture is negative and baby is asymptomatic.
\end{abstract}

Keywords: Antibiotics, Culture, Sepsis

\section{Background}

According to the World Health Organization (WHO) estimates there are about 5 million neonatal deaths a year globally, $98 \%$ of which occur in developing countries [1,2] accounting for about $26-34 \%$ of total deaths each year $[3,4,5]$. Despite substantial progress, the world has not achieved Millennium Development Goal (MDG) 4 that aimed to reduce child mortality by two thirds by 2015 .

Sepsis is the commonest cause of neonatal mortality; it is responsible for about $30-50 \%$ of the total neonatal

Manuscript received: $14^{\text {th }}$ July 2019

Reviewed: $24^{\text {th }}$ July 2019

Author Corrected: $30^{\text {th }}$ July 2019

Accepted for Publication: $5^{\text {th }}$ August 2019 deaths in developing countries. It is estimated that up to $20 \%$ of neonates develop sepsis and approximately $1 \%$ die of sepsis related causes [6]. Despite receiving extensive care in hospitals in the form of ventilator and inotropic support in intensive care set up, $2-3 \%$ of term and $20-30 \%$ of preterm infants still die of EOS $[7,8]$.

Complications associated with neonatal sepsis vary from necrotizing enterocolitis, meningitis, vision impairment, impaired head growth, functional disability in terms of difficulties in standing, locomotion eye-hand co-ordination or limb movement disorders that have long-term consequences for the neonates and the family. 


\section{Original Research Article}

Other very rare complications develop from sepsisassociated endocarditis and thrombosis and can include valvular damage, pulmonary embolism, and secondary infectious thrombo embolism $[9,10]$.

Up to50\% of these infants will suffer serious neurologic sequelae, including seizures, blindness, deafness or significant hearing loss, and cognitive delays in speech and language [11].

An untreated or inadequately treated case often means prolonged dependence on health care and this aggravates the situation especially in developing countries, where the load on health care due to the heavy patient load is already too much to bear.

In other words, Sepsis which can be prevented and treated, if missed ends up taking a toll in future on the patient, family and country resources.

As the signs and symptoms of the same can be very non-specific, "Suspected sepsis" is one of the common diagnosis made in neonatal care[12].

A high suspicion of sepsis is thus warranted and the various risk factors contributing to EOS such as LBW, maternal fever within 2 week of delivery, foul smelling liquor, PROM, unclean vaginal examination, prolonged labor and birth asphyxia are to be kept in mind to diagnose and treat EOS.

This further complicates the scenario for cliniciansfirstly to identify neonates with a high likelihood of sepsis promptly and initiate antimicrobial therapy; secondly to distinguish the "high-risk" healthyappearing infants or infants with clinical signs who do not require treatment; and lastly, but perhaps the most important being to decide the duration of antimicrobial therapy and when to discontinue it, once sepsis is deemed unlikely [13].

Clinical suspicion therefore frequently leads to empirical antibiotic therapy in uninfected infants.

The incidence of culture confirmed early-onset sepsis is rather low, around 0.4-0.8/1000 term infants in highincome countries.

6 to 16 times more infants receive therapy for culturenegative sepsis in the absence of a positive blood culture [14].
If the treatment of sepsis were completely benign, it would make no difference if every infant with the slightest chance of infection was treated. However, treating an uninfected infant for 5 to 7 days means disrupting maternal bonding and breast feeding for an extended period of time, pain and distress from starting IVs, exposing the infant to drugs with potential toxicities, fostering the development of antibiotic resistant flora, and increasing the probability that the infant will experience a more serious morbidity later in the course of hospitalization [15].

Recent data indicate that the perturbations of the nonresilient micro biota in early life may influence both the immune system and stem cell population negative impact on future health.

The extra costs of unnecessary administration of prolonged antibiotics, and later the cost of antibiotic resistance for the community along with collateral damage on their microbiodata will turn out to be a major hazard to community and health care,

if adequate steps are not taken for the rational use of antibiotics now [16].

Strict antibiotic policy and up-to-date guidelines will greatly impact management of neonatal sepsis today and the future.

Considering that majority of the centres still practice administration of antibiotics on clinical suspicion or empiric basis, there is great need for robust data and studies that clearly define sepsis and its antibiotic policy keeping in mind the concept of antibiotic stewardship.

Blood culture remains the gold standard for diagnosis of septicaemia and should be performed in all cases of suspected sepsis prior to starting antibiotics.

With obvious advantage of isolating the exact organism with its sensitivity profile.

It is now possible to detect bacterial growth within 1224 hours by using improved bacteriological techniques such as BACTEC and BACT/ ALERT blood culture systems.

These advanced techniques can detect bacteria at a concentration of 1-2 colony-forming units (CFU) per $\mathrm{ml}[17,18]$. 


\section{Original Research Article}

Studies that clearly define the outcome of early stoppage of antibiotics in blood culture negative term suspected early onset sepsis, is still lacking in Indian setup, considering which this study has been designed.

Aim and objective: 1. Safety of discontinuation of antibiotics after $48 \mathrm{hr}$ of culture negativity in suspected early onset neonatal sepsis in term neonates.

2. To study the Incidence of culture positivity in asymptomatic as well as symptomatic term babies with suspected early onset sepsis.

Material: The study cohort consisted of the all term babies hospitalised with early onset sepsis to the S.C.B M.C.H and S.V.P.P.G.I.P, Cuttack from October 2016 to September 2018.

After obtaining clearance from the institutional ethical committee, the patients fulfilling the inclusion criteria and not having exclusion criteria were enrolled into the study and were subjected to necessary investigations.

\section{Inclusion criteria}

1. Symptomatic babies $>37$ weeks of gestation with possible EOS

2. Gestational age $\geq 37$ weeks with suspected early onset sepsis.

\section{Exclusion criteria}

1.Neonates with suspected TORCH group of infection.

2.Any congenital anomaly.

3.Congenital heart disease.

4.Syndromic baby.

5.Any surgical conditions.

6.Neonates who have received antibiotics prior to hospitalisation.

A total number of 186 cases were included in the present study who had fulfilled the criteria.
Method: All the investigation were done in the central laboratory, department of pathology and biochemistry of S C B Medical College and SVP PG IP, Cuttack.

Using all aseptic precautions blood was collected from peripheral vein prior to starting antibiotic treatment.

\section{Investigations includes}

1. Complete blood count: This was performed by using Automated haematology analyzer MS4s and Pentra ES60 based on principle of Electric impedence.

2. CRP (Quantitative): estimated by Turbidimetric method using Prietest TOUCH analyser (robonik).

This method assesses agglutination of latex particles coated with antibody against CRP by quantifying the absorbed light (detection limit $>0.4 \mathrm{mg} / \mathrm{dL}$ ).,

3. I-T ratio: Peripheral smears for estimation of Immature: Total neutrophil (I:T) ratio, to see the morphology of neutrophils.

4. Micro ESR: Blood for micro ESR was directly collected in sodium-heparinised micro-hematocrit tubes.

5. Blood culture: Done by BacT/ALERT method.

6. Chest X-ray(if required)

7. The lumbar puncture was performed initially in all symptomatic babies and babies where sepsis is likely high, then subsequently in any baby with a positive blood culture or whose clinical course strongly suggests bacterial sepsis.

For babies who are critically ill and likely to have cardiovascular or respiratory compromise, lumbar puncture was deferred until the baby is stable.

Statistical analysis: Statistical analysis was done by statistical software SPSS for windows version 23. P values were calculated using chi-square test.

$\mathrm{P}<0.05$ was considered as significant and $\mathrm{P}<0.01$ as highly significant. 


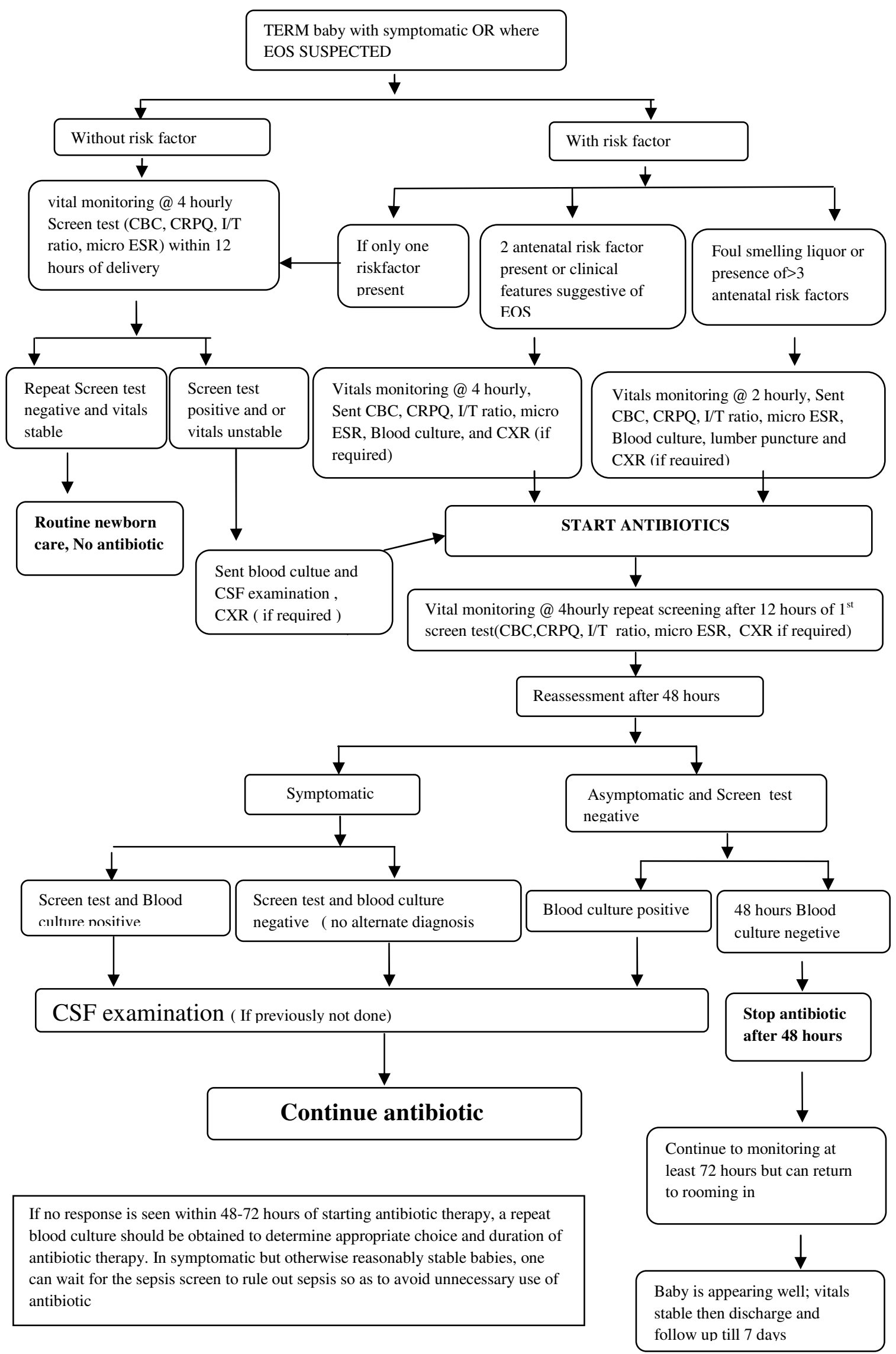

Figure 1: Practical approach plan of the study 
Enrolment

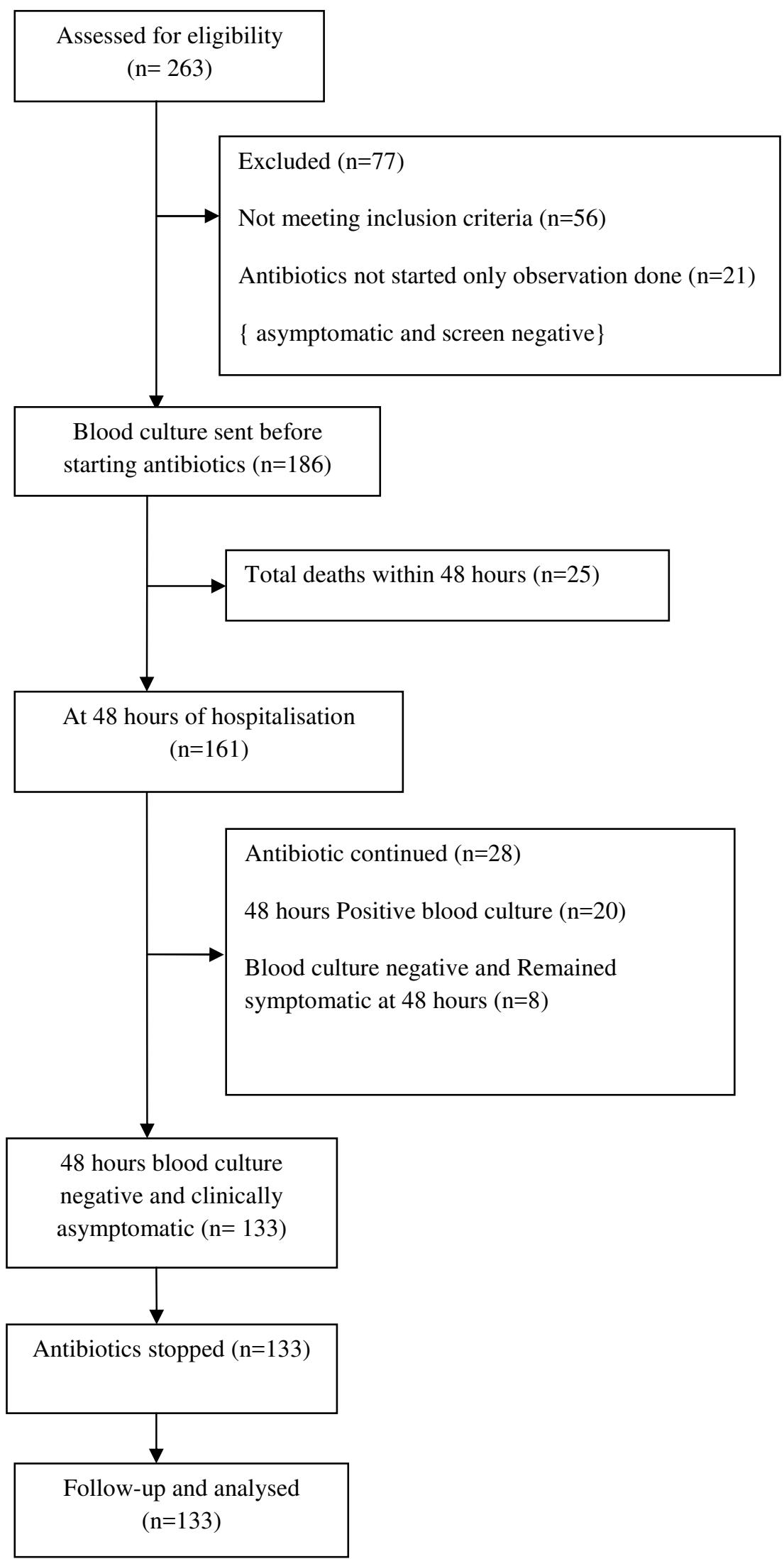

Figure 2: Flow diagram of the clinical study. 


\section{Results}

Table-1: Age distribution in the study group $(n=186)$.

\begin{tabular}{|c|c|c|}
\hline Age in hours & No. of cases & Symptomatic \\
\hline$<24$ hours & $75(40.3 \%)$ & $70(93.3 \%)$ \\
\hline $24-48$ hours & $52(28 \%)$ & $50(96 \%)$ \\
\hline $48-72$ hours & $39(21 \%)$ & $38(97.5 \%)$ \\
\hline $72-96$ hours & $20(10.8 \%)$ & $20(100 \%)$ \\
\hline Total & $\mathbf{1 8 6}(\mathbf{1 0 0} \%)$ & \\
\hline
\end{tabular}

Out of 186 cases 75 (40.3\%) presented within <24 hours of age, out of which, $93.3 \%$ were symptomatic; 52 (28\%) cases presented within 24 to 48 hours of age, of which $96 \%$ were symptomatic, and 39 (21\%) presented to 48 to 72 hours of life and rest $20(10.8 \%)$ were 72 to 96 hours of age at the time of presentation.

All babies who were subsequently culture positive presented within 24 hours of life.

Table-2: Sex distribution in the study group $(n=186)$

\begin{tabular}{|c|c|c|}
\hline Sex & No. of cases & Growth on blood culture \\
\hline Female & $67(36 \%)$ & $22(32.8 \%)$ \\
\hline Male & $119(64 \%)$ & $26(21.8 \%)$ \\
\hline Total & $\mathbf{1 8 6}(\mathbf{1 0 0} \%)$ & $\mathbf{4 8}(\mathbf{2 5 . 8 \%})$ \\
\hline
\end{tabular}

Out of 186 cases, the majority i.e. $119(64 \%)$ were male and $67(36 \%)$ were female.The male to female ratio in the present study was 1.8:1. Also, culture positive sepsis was seen more in males with the male to female ratio being $1.2: 1$.

Table-3: Distribution of cases with respect to birth weight

\begin{tabular}{|c|c|c|c|}
\hline Birth weight & No of cases & Symptomatic & Growth on blood culture (n=48) \\
\hline$<2.5 \mathrm{~kg}$ & $80(43 \%)$ & $75(93.7 \%)$ & $29(60 \%)$ \\
\hline$\geq 2.5 \mathrm{~kg}$ & $106(57 \%)$ & $103(97 \%)$ & $19(39.5 \%)$ \\
\hline Total & $\mathbf{1 8 6}(\mathbf{1 0 0} \%)$ & $\mathbf{1 7 8}$ & $\mathbf{4 8}(\mathbf{1 0 0} \%)$ \\
\hline
\end{tabular}

Out of 186 cases, $106(57 \%)$ were normal birth weight $(\geq 2.5 \mathrm{~kg})$ and $80(43.2 \%)$ were low birth weight. 75 (93.7\%) babies out of 80 cases of the LBW babies were symptomatic $.19(67.8 \%)$ of 28 died subsequently.

Among the 48 culture positive babies, 29 (60\%) were Low birth weight.

Table-4: Initial assessment at the time of admission $(n=186)$

\begin{tabular}{|c|c|}
\hline Initial assessment & No of cases \\
\hline Symptomatic & $178(95.7 \%)$ \\
\hline Asymptomatic & $8(4.3 \%)$ \\
\hline Total & $\mathbf{1 8 6}(\mathbf{1 0 0} \%)$ \\
\hline
\end{tabular}

Out of 186, $178(95.7 \%)$ were symptomatic and $8(4.3 \%)$ were asymptomatic at the time of admission.

Table-5: Relation of death rate with day of hospitalisation

\begin{tabular}{|c|c|}
\hline Day of hospitalisation & Death $(\mathbf{n = 2 8})$ \\
\hline Day 1 & $19(67.8 \%)$ \\
\hline Day 2 & $6(21.4 \%)$ \\
\hline Day 3 & $3(10.7 \%)$ \\
\hline Total & $\mathbf{2 8}(\mathbf{1 0 0} \%)$ \\
\hline
\end{tabular}




\section{Original Research Article}

Maximum death occurred on day 1 of life i.e. 19 (67.8\%), on day 26 (21.4\%) and on day 3, 3 (10.7\%).Mortality of culture positive sepsis was found to be $58.4 \%$ (28 deaths out of 48 culture positive).

Table-6: Incidence of perinatal risk factor among suspected case of early onset sepsis (n=186)

\begin{tabular}{|l|c|c|}
\hline Risk factors & No of cases & P value \\
\hline Low birth weight & $80(43 \%)$ & 0.004 \\
\hline Prolonged rupture of membrane & $37(19.9 \%)$ & 0.002 \\
\hline Foul smelling liquor & $16(8.6 \%)$ & 0.086 \\
\hline Unclean vaginal examination & $8(4.3 \%)$ & 0.112 \\
\hline Prolonged labour & $9(4.8 \%)$ & 1.71 \\
\hline Maternal fever & $2(1.1 \%)$ & 0.43 \\
\hline Perinatal asphyxia & $45(24.2 \%)$ & 3.65 \\
\hline
\end{tabular}

$P$ value $<.05$, chi-square test applied

Among various risk factors studied in relation to suspected early onset sepsis, low birth weight led the list in the present study with $80(43 \%)$ cases, followed by perinatal asphyxia 45(24.2\%) and prolonged rupture of membranes $37(19.9 \%)$.

A significant association was noted between the incidence of blood culture positivity with 2 risk factors namely, low birth weight and prolonged rupture of membranes $(\mathrm{p}<0.05)$.

Table-7: Number of perinatal risk factors present at the time of admission $(n=186)$

\begin{tabular}{|c|c|c|}
\hline No of risk factor & No of cases & Growth on blood culture \\
\hline 0 & $64(34.4 \%)$ & $11(17.2 \%)$ \\
\hline 1 & $77(41.3 \%)$ & $15(19.5 \%)$ \\
\hline 2 & $34(18.3 \%)$ & $14(41.2 \%)$ \\
\hline$\geq 3$ & $11(5.9 \%)$ & $8(72.7 \%)$ \\
\hline Foul smelling liquor & $16(8.6 \%)$ & $7(43.8 \%)$ \\
\hline Total & $\mathbf{1 8 6}(\mathbf{1 0 0} \%)$ & \\
\hline
\end{tabular}

Among 186 cases, 64 (34.4\%) had no perinatal risk factors at the time of admission where as 77(41.3\%) had single risk factor. 2 risk factors were noted in $34(18.3 \%)$ of the study population, $>2$ risk factor $44(23.7 \%), \geq 3$ risk factors were seen in $11(5.9 \%)$ of the cases. However, $16(8.6 \%)$ babies had foul smelling liquor.

Table-8: Assessment of risk factors at the time of admission $(n=186)$

\begin{tabular}{|c|c|c|}
\hline No. of risk factor & Asymptomatic & Symptomatic \\
\hline $0(n=64)$ & 0 & $64(100 \%)$ \\
\hline $1(n=77)$ & 0 & $77(100 \%)$ \\
\hline $2(n=34)$ & $5(14.7 \%)$ & $29(85.3 \%)$ \\
\hline$\geq 3(n=11)$ or FSL & $3(27.3 \%)$ & $8(72.7 \%)$ \\
\hline Total $(\mathbf{n}=\mathbf{1 8 6})$ & $\mathbf{8}(\mathbf{4 . 3 \%})$ & $\mathbf{1 7 8}(\mathbf{9 5 . 7 \% )}$ \\
\hline
\end{tabular}

Out of the 64 cases which had nil perinatal risk factor, all were symptomatic at admission. Similarly, among 77 cases with single risk factor, all were symptomatic. Also, among 34 cases with 2 risk factor, 29 (85.3\%) were symptomatic and $5(14.7 \%)$ were asymptomatic but sepsis screen positive.

Further, $8(72.7 \%)$ out of 11 cases which had $\geq 3$ risk factors, were symptomatic. 
Table-9: Perinatal risk factors at admission versus sepsis screen result.

\begin{tabular}{|c|c|c|c|c|c|}
\hline \multirow{2}{*}{ Risk factors } & \multicolumn{4}{|c|}{ Sepsis screen } & \multirow{2}{*}{ P Value } \\
\hline & Result & Initial & p value & After 48 hours & \\
\hline \multirow{2}{*}{$\begin{array}{c}<2 \text { Risk factor } \\
\quad(n=141)\end{array}$} & No growth & $106(75.2 \%)$ & \multirow{4}{*}{0.05} & $115(81.5 \%)$ & \multirow{4}{*}{0.001} \\
\hline & Growth & $35(24.8 \%)$ & & $26(18.5 \%)$ & \\
\hline \multirow{2}{*}{$\begin{array}{l}\geq 2 \text { Risk factors } \\
\quad(\mathrm{n}=45)\end{array}$} & No growth & $27(60 \%)$ & & $23(51 \%)$ & \\
\hline & Growth & $18(40 \%)$ & & $22(49 \%)$ & \\
\hline
\end{tabular}

Out of 141 cases with $<2$ risk factors, $35(24.8 \%)$ had positive initial sepsis screen and all were symptomatic at the time of admission. After 48 hours $26(18.5 \%)$ showed growth on blood culture. All were symptomatic at the time of admission.

Table-10: Assessment of sepsis screen prior to starting antibiotics $(n=186)$ and after 48 hours.

\begin{tabular}{|c|c|c|c|c|c|}
\hline \multirow[b]{2}{*}{ Assessment } & \multicolumn{4}{|c|}{ Sepsis screen } & \multirow[b]{2}{*}{ P Value } \\
\hline & Result & Initial (178/8) & P Value & $\begin{array}{l}\text { After } 48 \text { hours } \\
(22 / 139)\end{array}$ & \\
\hline \multirow{2}{*}{ Symptomatic } & No growth & $50(28 \%)$ & \multirow{4}{*}{0.51} & $17(77.2 \%)$ & \multirow{4}{*}{0.001} \\
\hline & Growth & $128(72 \%)$ & & $5(22.7 \%)$ & \\
\hline \multirow{2}{*}{ Asymptomatic } & No growth & $3(37.5 \%)$ & & $6(4.4 \%)$ & \\
\hline & Growth & $5(62.5 \%)$ & & $133(95.6 \%)$ & \\
\hline
\end{tabular}

Out of 186 cases, $178(95.7 \%)$ cases were symptomatic out of which 50 (28\%) cases had positive sepsis screen. Further, $8(4.3 \%)$ cases were asymptomatic out of which $3(37.5 \%)$ cases had positive screen.

Out of 161 cases, 22 (13.7\%) cases remained symptomatic out of which 17 (77\%) had growth on blood culture.However, $139(86.3 \%)$ cases remained asymptomatic out of which $6(4.4 \%)$ had growth on blood culture.

\section{Discussion}

The given study was conducted in the tertiary care hospital setting in Cuttack, and studied the demographic profile of those neonates identified as suspected sepsis, their associated risk factors, and the further attempted to assess the need to continue antibiotics based on the results of blood culture. However, there is no data available regarding the safety of such practice.

Adding to this, the study further acts like a prototype of the situation in a developing country as ours where resources are limited as compared to the huge load of patients, and limited availability of facilities round the clock, which further complicates the diagnosis and management of neonatal sepsis. In most situations, babies presenting with symptoms or with risk factors land up getting prolonged course of antibiotics.

Many a time use of antibiotics happen without any blood culture so only the baby's symptoms guide the duration of antibiotics. The present study tried to utilise the BacT/Alert blood culture to diagnose cases of culture positive sepsis and rationalise use of antibiotics.
Out of 186 cases, most of the neonates presented within 24 hours of life $(40.3 \%)$ and there were significant number of babies, who presented by 48 hours, who reached the hospital late after onset of symptoms, perhaps due to distance from the tertiary centre or were referred from periphery.

Polin R A (2012)[13] also had similar findings according to which most cases (80\% to $90 \%$ ) of EOS presented in the first 24 to $48 \mathrm{~h}$ of life.

Kari A et al (2014)[19] further showed that most neonates developed signs of EOS within 12 hours of life, which was also on the lines of our findings.

Male babies were predominant in the present study, the male to female Ratio being 1.8:1. Similar finding was seen in the study done by A.c. Buch et al[20], where Male to Female ratio was 1.8: 1. Morven $\mathrm{S}$ and colleagues [21] observed male to female ratio of $2: 1$, which again is consistent with the present study. Males outnumbered females in numerous studies, in terms of 


\section{Original Research Article}

EOS cases, including those done by Chandra 1988[22], Antoniette 2005[23], Rajarshi Basu et al (1.5:1)[24] and Heena Rihan et al (1.63:1)[25]. In the present study, Early onset culture positive neonatal sepsis was more common among male babies as well (male: female ratio 1.2:1). The increased incidence of sepsis in male newborns has been could be due to presence of only single $\mathrm{X}$-chromosome in them, as opposed to two in female. In the study done by Chandra [22]in 1998, he mentioned that the factors regulating the synthesis of gamma globulin are situated on X-chromosome, which makes male inherently more prone for sepsis.

Study conducted by K. Padma Malini et al (2016)[26], 112 male babies $(56 \%)$ and 88 female babies (44\%) were affected by neonatal septicemia. Preponderance was also seen in several other studies by Piyush Gupta et al [27], Sumon et al[28], Philip et al[29], Khatua, et al[30], Sinha, et al[31] observed that the male to female ratio was 1.7. The situation in developing countries is however more complex and influenced by a multitude of factors, ranging from cultural beliefs and traditions to availability of nearby health facility. There still exists an unspoken bias to towards the male child, thus causing more males to be brought to focus of medical care, and more so in a higher centre. On the other hand, there is delayed health seeking for female babies. These inequalities further add to the final male to female ratio of neonatal sepsis.

Out of 186 cases, who were suspected to have sepsis in the present study, $80(43.2 \%)$ were low birth weight and $60 \%$ babies who are culture positive were LBW and both the above incidences are higher than the incidence of LBW in general population. This shows LBW babies are at increased risk of sepsis and other neonatal illness with clinical symptoms similar to sepsis. Early-onset septicemia was present in $58.8 \%$ with normal birth weight $\geq 2000 \mathrm{gm}$ in a study done by Jyoti Bendigeri et al (2015)[32], which is consistent with result of the present study.

However, various studies done by Gott of 1992 [17] Kumar 1994, Rajarshi Basu et al 2014[24] and Heena et al[25]2016 showed majority of cases of proven sepsis to be in premature and low birth weight $(65 \%)$. A.C. Buch et al (2011)[20] also reported that the $80.8 \%$ neonates affected with sepsis were low birth weight. This was attributed to immature cellular and humoral immune system at birth, including phagocytic activity, decrease response to localize infection etc., making them vulnerable to infections [23]. Further it is postulated by Bhakoo [18] that placental transport of $\operatorname{IgG}$ from maternal to fetal circulation is hampered in SGA neonates, due to presence of placental insufficiency. The difference in the present study could probably be due to consideration of suspected sepsis cases that are exclusively term, in contrast to other studies where sepsis has been studied in both low birth weight and premature babies, suggesting that preterm may act as a confounding factor, for early onset sepsis. 178 (95.7\%) were symptomatic at presentation and $8(4.3 \%)$ were asymptomatic. Out of total number of deaths maximum death happened on day 1 of hospitalisation i.e. 19 $(67.8 \%)$; on day 2, 6 deaths $(21.4 \%)$ and on day 3,3 deaths $(10.7 \%)$. This is because the babies may be very sick at the time of admission and that could be due to delayed initiation of treatment due to poor health seeking behaviour by parents or delay in referral.

Mortality rate of culture positive sepsis was found to be $58.4 \%$ and $67.8 \%$ death happened within 24 hours of hospitalisation. There was no single death on blood culture negative babies. This high mortality in culture positive babies could be due to delay in referral, antibiotic initiation and poor transport. Mathur et al [33], who observed mortality of $64.5 \%$ when the onset of illness was early. High mortality in association with early-onset septicaemia was reported by Bhakoo et al [18] as well.

However, A. C. Buch [20] reported that the mortality rate of $24.61 \%$ in culture positive cases, which is quite less than ours and $18.18 \%$ death in culture negative cases which is higher than the present study. As per DeNIS [34] reported a lesser mortality rate of $8.6 \%$ in culture positive sepsis in intramural babies. The difference of the mortality is because all the babies were intramural and diagnosis and initiation of antibiotic was quick.

Among various risk factors studied in relation to suspected early onset sepsis, low birth weight led the list in the present study, with $80(43 \%)$ cases, followed by perinatal asphyxia $45(24.2 \%)$ and prolonged rupture of membranes $37(19.9 \%)$. Other risk factors noted included foul-smelling liquor $8.6 \%$, unclean vaginal examination $4.3 \%$, prolonged labour $4.8 \%$, and maternal fever $1.1 \%$. The above results were similar to those seen in study conducted by Meenusingh et al[35] where majority of suspected sepsis cases had prolonged rupture of membrane(45\%), birth asphyxia (34\%), and low birth weight $(60 \%)$ as their risk factors. Further foul-smelling liquor, maternal fever, unclean vaginal examination and birth asphyxia were proven to be independent, and PROM and prolonged labour as 


\section{Original Research Article}

dependent factors. The study further mentioned few combinations of risk factors such as LBW (32\%), PROM+PL (14\%), PROM+BA (35\%) and PROM+FSL $(70 \%)$ to contribute to EOS, rather than each factor individually. Study done by Mamta Jajoo et al[36]2018 reported low birth weight as most common risk factor in EOS (68.3\%), followed by perinatal asphyxia and multiple vaginal examination (35.4\%). A study done on risk factors in neonatal sepsis by Agarwal et al[37] showed birth asphyxia as one of the most common risk factors associated with EOS.

Kishore et al [38] reported $19.2 \%$ of vertical transmission and sepsis in babies born to mothers with prolonged rupture of membrane. Anand et al [39] observed $29.3 \%$ of EOS can be attributed to prolonged rupture of membrane. These findings are consistent with the present study. Further, N. Mehrotra [40] noted threefold increase in incidence of sepsis after PROM. Investigators of the Delhi Neonatal Infection Study (DeNIS 2016) collaboration[34]reported that Maternal fever within 7 days before delivery $(7.8 \%)$, Per-vaginal examination $(\geq 3) \quad(38.5 \%)$, Prolonged rupture of membranes (18 h or more) $(14.5 \%)$, Prolonged labour (24 h or more) (1.3\%), Foul-smelling liquor $(2.8 \%)$ are the major risk factors contributing to neonatal EOS, the findings of which are in contrast to the present study.

$64(34.4 \%)$ had no perinatal risk factors at the time of admission where as $77(41.3 \%)$ had single risk factor. 2 risk factors were noted in $34(18.3 \%)$ of the study population, $\geq 3$ risk factors were seen in $11(5.9 \%)$ of the cases, however $16(8.6 \%)$ babies had foul smelling liquor. In a study done by Vamsi Krishna kondle et al [41] on septicaemia in neonates, around $14 \%$ of cases had no risk factors, as compared to $34 \%$ in the present study. The difference could be due to difference in the type of population group. Single risk factor was present in $41 \%$ of our cases, which was much higher than that observed by Meenu Singh et al [35], where 15\% of babies had single risk factor. Foul smelling liquor was present in $8.6 \%$ of our population as compared to Meenu singh et al [35], where it was 13\%.

Unlike the study done by Meenu Singh et al [35] where the incidence of 2 or more risk factors is as high as $85 \%$, in the present study it formed only $24 \%$ of the population. Among those, 2 risk factors were seen in $18 \%$ and three or more than three was present in $6 \%$ of the Suspected EOS. The difference could probably be because of the difference in the structure of the former study, which was a case control study and perinatal history was obtained from the obstetric team rather than mothers. On comparing the number of risk factors present at the time of admission with the presence or absence of symptoms at admission, showed that the number of risk factors did not correlate with presence of symptoms at admission. In fact, all those babies with nil or singe risk factor were symptomatic [Table 10]. Thus, presence of symptoms should be assessed independently than the number of risk factors.

In the present study, the above sepsis screen was performed at admission and repeat screen was done in those patients in whom first screen was negative, but baby clinically symptomatic, or first screen obtained before 12 hours of life (10 babies had repeat screen positive) prior to starting antibiotics in all 186 suspected EOS cases. Overall 34\% symptomatic babies were screen positive. However, $37 \%$ of asymptomatic babies were also sepsis screen positive.

Out of 186 cases, 48 (25.8\%) showed growth in blood culture media, in which blood was drawn prior to starting antibiotics. The finding was consistent with the result obtained by Sharma 1993[42], Lee 1997[43], Joshi at al 2000[44], NNPD 2002-03[45],Hussein 2007[46], Dr. G. Vandana 2017[47] and Mamtajajoo et al 2018[36] where the culture positivity was $20 \%, 41 \%$, $25 \%, 28.6 \%, 42 \%, 42 \%, 22 \%$ and $18 \%$ respectively.

All babies who are culture positive were symptomatic at presentation. Out of 161 cases, $22(13.7 \%)$ cases remained symptomatic out of which 17 (77\%) had growth on blood culture. $49 \%$ of neonates with positive sepsis screen had growth on blood culture, where as $16.5 \%$ of those with sepsis screen negative had growth in blood culture. This shows sepsis screen is neither $100 \%$ sensitive or specific. The limitation of the present study was smaller size of study and the study was a single centre study.

Summary- Out of total 186 term neonates with suspected early onset sepsis, majority were male (64\%) and mostly presented in $1^{\text {st }} 24$ hours of life (40\%). 43\% of cases were low birth weight. The most common perinatal risk factor noted in the present study was Low birth weight $(43 \%)$ followed by perinatal asphyxia (24.2\%). PROM was found in $20 \%$ babies. $96.2 \%$ of the neonates were symptomatic at the time of admission; the most common symptom being respiratory distress $(73.6 \%)$.The blood culture was found to be positive in $25.8 \%$ of the study population. The incidence of culture positive sepsis in symptomatic babies was $27 \%$ which emphasises that all babies with symptoms are not sepsis. No asymptomatic babies had culture positive 


\section{Original Research Article}

sepsis. $28.4 \%$ had their initial sepsis screen positive. $49 \%$ of those neonates with screen positive had growth on blood culture.Sensitivity and specificity of sepsis screen was $54 \%$ and $80 \%$ with predictive value of positive test $49 \%$ and predictive value of negative test $83.5 \%$. Sepsis screen done at admission (with repeated screen at 12 hours) has limited value in identifying definite sepsis in EOS.Antibiotics were stopped in 133 babies who were clinically asymptomatic and blood culture negative at 48 hours into antibiotics initiation. The unnecessary use of antibiotics was cut down by $82.6 \%$. No adverse outcome suggestive of sepsis was noted in the follow up period of 7 days of the neonates in whom antibiotics were stopped.

\section{Conclusion}

Sepsis is one of the major cause of neonatal mortality. So, there is an inherent urgency in initiating antibiotics in babies born with multiple perinatal risk factors for sepsis, with or without clinical symptoms. However the signs and symptoms of sepsis are very non specific. Thus, many babies who are not having infection or symptomatic because of non infectious causes, receive antibiotics for a long duration. This increases the length and cost of hospitalisation, increases parental anxiety, creates an unnecessary burden to the over burdened health system and overall, increases antibiotic resistance with its consequences.

With the present study, it was observed that along with evaluation of clinical condition of the baby and 48 hour BacT/Alert blood culture report, antibiotics can be safely discontinued in those babies who are clinically asymptomatic and 48 hour blood culture negative. Early onset sepsis has vague clinical presentation for which empirical antibiotics is practiced universally without knowing the blood culture status.

\section{What the present study adds to the existing knowledge?}

This study provides a means to reduce the burden and antibiotic misuse by establishing safety in discontinuing antibiotics in term neonates with suspected early onset sepsis with negative blood culture at 48 hours and clinically asymptomatic.

\section{Author's contribution}

Dr. Manas Ranjan Upadhyay: Concept and design of design, revising critically for intellectual contents, final approval and agreement to be accountable.
Dr. Kiran Chandra Pankaj: Acquisition, analysis and data interpretation, drafting the work, final approval and agreement to be accountable

Dr. Sristi Ganguly: Data analysis and interpretation, drafting the work, final approval and agreement to be accountable

Dr. Mangal Charan Murmu: Manuscript writing, validation of data,final approval and agreement to be accountable

Funding: Nil, Conflict of interest: None initiated, Permission from IRB: Yes

\section{References}

1. Neonatal and Perinatal Mortality. WHO/FRH MSM/ 967 Geneva;1996.(https://apps.who.int/iris/ bitstream/ 10665/43444/1/9241563206_eng.pdf)

2. Stoll BJ. The global impact of neonatal infection. Clin Perinatol. 1997;24(1):1-21.doi: https://doi.org/ 10. 1016/S0095-5108(18)30181-7

3. Vergnano S, Sharland M, Kazembe P, Wansambo CM, Heath PT. Neonatal sepsis. An international perspective. Arch. Dis. Child. Fetal Neonatal Ed., 2005; 90 (3): F220- F224. doi: 10.1136/adc.2002.022863

4. Lawn JE, Cousens S, Zupan J; Lancet Neonatal Survival Steering Team 4 million neonatal deaths: when? Where? Why? Lancet. 2005;365(9462):891-900. doi: https://doi.org/10.1016/S0140-6736(05)71048-5

5. Thaver D, Zaidi AK. Burden of neonatal infections in developing countries: a review of evidence from community-based studies. Pediatr Infect Dis J. 2009; 28 (1 Suppl):S3-S9.doi: 10.1097/INF.0b013e 3181958755.

6. Bang AT, Bang RA, Baitule SB, Reddy MH, Deshmukh MD Effect of home-based neonatal care and management of sepsis on neonatal mortality: field trial in rural India. Lancet. 1999;354(9194):1955-1961. doi: https://doi.org/10.1016/S0140-6736(99)03046-9

7. Stoll BJ, Hansen NI, Sánchez PJ, Faix RG, Poindexter BB, Van Meurs KP, et al; Early onset neonatal sepsis: the burden of group B Streptococcal and E. coli disease continues. Pediat. 2011;127(5):81726.doi: 10.1542/peds.2010-2217.

8. Phares CR, Lynfield R, Farley MM, Mohle-Boetani $\mathrm{J}$, Harrison LH, Petit S, et al; Epidemiology of invasive group B streptococcal disease in the United States, 1999-2005. JAMA. 2008;299(17):2056-2065. doi: 10. 1001/ jama.299.17.2056. 


\section{Original Research Article}

9. Gordon AL, English M, Tumaini Dzombo J, Karisa M, Newton CR. Neurological and developmental outcome of neonatal jaundice and sepsis in rural Kenya. Trop Med Int Health. 2005;10(11):1114-20.doi: https:// doi. org/10.1111/j.1365-3156.2005.01496.x

10. Stoll BJ, Hansen NI, Adams-Chapman I, Fanaroff AA, Hintz SR, Vohr B, Higgins RD; Neurodevelopmental and growth impairment among extremely lowbirth-weight infants with neonatal infection. JAMA. 2004;292(19):2357-65. doi:10.1001/ jama. 292.19.2357

11. Long SS, Pickering LK, Prober CG. Principles and practice of pediatric infectious disease. Elsevier Health Sciences; 4th ED 2012. PP-707-712.

12. Escobar GJ, The Neonatal "Sepsis Work-up": Personal Reflections on the Development of an Evidence- Based Approach Toward Newborn Infections in a Managed Care Organization. Pediat. 1999;103(1): 360-373.

13. Polin RA; Committee on Fetus and Newborn. Management of neonates with suspected or proven early-onset bacterial sepsis. Pediatrics. 2012;129(5): 1006-15. doi: 10.1542/peds.2012-0541.

14. Klingenberg C, Kornelisse RF, Buonocore G, Maier RF, Stocker M. Culture-negative early-onset neonatal sepsis-at the crossroad between efficient sepsis care and antimicrobial stewardship. Front. Pediatr. 2018;6:285. doi: 10.3389/fped.2018.00285

15. Schulfer A, Blaser MJ. Risksof AntibioticExposures EarlyinLifeontheDevelopingMicrobiome. PLoS Pathog. 2015;11(7):e1004903.doi:10.1371/journal.ppat. 100490.

16. Gerdes JS, Polin R. Early diagnosis and treatment of neonatal sepsis. Indian J Pediatr 1998;65(1):63-78.doi: https://doi.org/10.1007/BF02849696

17. Gotoff SP, Behrman RE. Neonatal septicemia. J. Paed. 1970:76(1):142-153.

18. Bhakoo ON. Neonatal bacterial infections at Chandigarh-A decade of experience. Indian J Pediatr 1980; 47(5):419-424.doi: https://doi.org/10.1007/BF 02759845

19. Kari A. Simonsen, Ann L. Anderson-Berry, Shirley F. Delair, H. Dele Davies . Early-Onset Neonatal Sepsis doi:10.1128/CMR.00031-13.
20. Buch A, Srivastava, Kumar H, Jadhav P. Evaluation of hematological profile in early diagnosis of clinically suspected cases of neonatal sepsis. Int J BAMS. 2011; $1(1): 1-6$.

21. Edwards M. Postnatal Bacterial Infections. In: Martin RJ, Fanaroff AA, Walsh MC, editors. Fanaroff and Martin's neonatal-perinatal medicine: diseases of the fetus and infant. $9^{\text {th }}$ ed. Philadelphia: Saunders/ Elsevier; 2011. pp. 793-830.

22. Chandra A, Rao MN, Srinivas M and Shyamala S. Rapid diagnostic tests in neonatal septicemia. Indian J Pediatr.1988;55(6):947-953. doi:10.1007/bf02727835

23.Antoinette BMW and Flora DIP. Clinical correlation of neonatal and maternal haematological parameters as predictors of neonatal sepsis. Pediatric Infect Dis Soc Philippines J. 2005;9;(2):36-43.

24. RajarshiBasu, Shyamal Kumar Bandyopadhyay, Study on Correlation between Sepsis Screening and Blood Culture in Neonatal Sepsis; IOSR-JDMS. 2014; 13(5):52-56.

25. Hassan HR, Gohil JR, Desai R, Mehta RR, Chaudhary VP. Correlation of blood culture results with the sepsis score and sepsis screen in the diagnosis of early-onset neonatal septicemia. J Clinic Neonatol. 2016;5(3):193-198. DOI: 10.4103/2249-4847.191263

26. K. Padma Malini, Sunethri Padma, N. Srivani, Chaitanya Kumari, O. Shravan Kumar, J. Venkateswara Rao.Application and correlation of hematological scoring system and serum prolactin levels in early diagnosis of neonatal sepsis - 3 year study. IAIM. 2016; 3 (11):36-45.

27. Piyush G, Murali MV, Faridi MMA, Caul PP, Ramachandran VG, V Talwar. Clinical profile of Klebsiella septicemia in neonates. Indian JPediat. 1993; 60 (4):565-572.

28. Shetty SMV, Moses GL, Subramanian L and Balagopal RV. A critical analysis of septicemia in infancy. Indian J Paediatr. 1976;13;(1):443-446.

29. Philip Alistair GS, Hewitt JR. Early diagnosis of neonatal sepsis. Pediatr 1980;65(5):1036-1041.

30.KhatuaSP, Das AK, Chatterjee BD, Khatua S, Ghose B, Saha A. Neonatal septicemia. Indian J Pediatr. 1986; 53(4):509-514.doi:https://doi.org/10.1007/BF027 49537 


\section{Original Research Article}

31. Sinha N, Deb A, Mukherjee AK. Septicemia in neonate and early infancy. Indian J Pediatr. 1986;53 (2):249-256.doi: https://doi.org/10.1007/BF02748516

32. Patil M, Bendigeri J. Clinico-epidemiological study of Acne Vulgaris in Southern India. Int J Biomed Res. 2015;6(07):509-511. 509. doi: 10.7439/ijbr

33. Mathur NB, Khalil A, Sarkar R, Puri KK. Mortality in neonatal septicemia with involvement of mother in management. Indian Pediatr. 1991;28(11):1259-1263.

34. Investigators of the Delhi Neonatal Infection Study (DeNIS) collaboration. Characterisation and antimicrobial resistance of sepsis pathogens in neonates born in tertiary care centres in Delhi, India: a cohort study; Lancet Glob Health 2016; 4: e752-60. doi: 10.1016/S2214-109X(16)30148-6.

35. Singh M, Narang A, Bhakoo ON. Predictive perinatal score in the diagnosis of neonatal sepsis. $\mathrm{J}$ Trop Pediatr. 1994;40(6):365-8.doi: 10.1093/tropej/ 40.6.365.

36. Jajoo M, Kapoor K, Garg LK, Manchanda V, Mittal SK.To Study the Incidence and Risk Factors of Early Onset Neonatal Sepsis in an Out born Neonatal Intensive Care Unit of India. J Clinic Neonatol. 2015; 4(2):91-95. doi: 10.4103/2249-4847.154106

37. Agarwal M, Chathurvedi P, Dey SK, Narang P, Coagulase negative staphylococcal septicemia in newborn. Indian Pediatr. 1990;27(2):163-169.

38. Kishore K, Deorari AK, Singh M, Bhujwala RA. Early onset neonatal sepsis: Vertical transmission from maternal genital tract. Indian Pediatr. 1987; 24(1):45-8.
39. Anand NK, Gupta AK, Mohan M,Lamba IMS, Gupta R, Shrivastava L.Coagulase negative septicemia in newborn. Indian Pediatr. 1991;28:1241-1248.

40. Mehrotra N, Kumar A, Chansoria M, Kaul KK. Neonatal sepsis, correlation of maternal and neonatal factors to positive blood. cultures. Indian Pediatr 1985; 22 (4):275-280.

41. Kondle KV, T. Clement Manohar, Clinical study of neonatal septicemiawith reference to early indicators of sepsis in NICU, PIMS. PediatrEducat Res. 2017;5 (3):197-202.

42. Sharma A, Krishna Kutty CV, Sabharwal U, Sushila and Mohan H (1993). Evaluation of sepsis screen for diagnosis of neonatal septicemia. Indian J Pediatr. 1993; 60(4):559-563.

43. Lee YH, Kim SW, Kim MH, Choi YS. Predictive factors and its usefulness in early diagnosis of neonatal sepsis. J Korean Soc Neonatol. 1997;4(2):195-204.

44. Joshi SG, Ghole VS, Niphadkar KB. Neonatal Gram-Negative Bacteremia. Indian J Pediatr 2000;67 (1):27-32.doi: https://doi.org/10.1007/ BF02802632

45. NNPD Report of the National Neonatal Perinatal Database (National Neonatology Forum) 2002-03.

46. Hussein AB, Khaled MAR. CRP in neonate with suspected septicemia. Rawal Med J. 2007;32(1):24-27.

47. Vandana G, Magar LR, Praveen, Devi K. Haematological Profile in Neonatal Septiceamia; IOSRJDMS. 2017; 16(4): 11-17. doi: 10.9790 /08531604091117

\section{How to cite this article?}

Upadhyay M.R, Pankaj K.C, Ganguly S, Murmu M.C. Early stoppage of antibiotics in blood culture negative term suspected early onset sepsis and their 7 days outcome. Int J Pediatr Res. 2019; 6(08):388-400.doi:10.17511/ijpr. 2019.i08.02 